\title{
Posterior mandibulada dental implant cerrahisi sırasında lingual kemik perforasyon riskinin değerlendirilmesi: 3 Boyutlu implant planlama programı kullanılarak yapılan retrospektif çalışma
}

\author{
Murat Ulu(0000-0002-3338-7198) ${ }^{\alpha}$, Furkan CIclk(0000-0002-9482-5761) ${ }^{\beta}$, Fahrettin Kalabalık(0000-0001-7084-4995) ${ }^{\nu}$, \\ Keremcan Kuru(0000-0002-1118-3274) ${ }^{\alpha}$, Hüseyin Akçay(0000-0001-7730-8212) ${ }^{\alpha}$, Şükrü Enhoş(0000-0002-8558-4951) ${ }^{\lambda}$
}

Selcuk Dent J, 2018; 5: 233-238 (Doi: 10.15311/selcukdentj. 385564)

Bașvuru Tarihi: 29 Ocak 2018 Yayına Kabul Tarihi: 23 Mayıs 2018

Öz

Posterior mandibulada dental implant cerrahisi sırasında lingual kemik perforasyon riskinin değerlendirilmesi: 3 Boyutlu implant planlama programı kullanılarak yapılan retrospektif çalışma

Amaç: Bu çalışmanın amacı dişsiz mandibular molar ve premolar bölgelere bilgisayar ortamında planlama programı kullanılarak konik ışınlı bilgisayarlı tomografi (KIBT) görüntüleri üzerinde yerleştirilen implantların sebep olduğu lingual perforasyon prevalansının değerlendirilmesi ve kretin morfolojik yapısı ile lingual kortikal kemik perforasyon riski arasındaki ilişkinin belirlenmesidir.

Gereç ve Yöntemler: İzmir Katip Çelebi Üniversitesi Diş Hekimliği Fakültesi arşivindeki KIBT görüntüsü alınmış hastalardan 543'ünün tomografi görüntüleri tarandı ve dahil edilme kriterlerine uyan 107 hastanın görüntüleri üzerinde, Simplant ${ }^{\circledR} \quad$ implant planlama programı kullanılarak 179 adet sanal implant yerleştirildi. Kretler kesitlerine göre $P$ tipi, $C$ tipi ve $U$ tipi olmak üzere 3 gruba ayrıldı. Panoramik radyografide inferior alveolar kanala $2 \mathrm{~mm}$ uzaklıkta olacak şekilde uzunlukları hesaplanan dental implantların lingual kemikle olan ilişkisi değerlendirildi.

Bulgular: Lingualde oluşan perforasyonların büyük çoğunluğu U tipi kretlerde $(\% 87,5)$ görülürken, perforasyon riskinin $8 \mathrm{~mm}$ uzunluktan sonra arttığı görülmüştür. C tipi kretlerde dört $(\% 8,3)$ implantda perforasyona rastlanırken, $P$ tipi kretlerde $14 \mathrm{~mm}$ uzunlukta sadece iki (\% 4,2) hastada lingual perforasyon görülmüştür.

Sonuç: Bu çalışmanın sonuçları implant uygulamalarında, osteotomi yapılacak alanın anatomik oluşumlara olan komşuluğunu değerlendirmede KIBT'nin değerini bir kez daha ortaya koymaktadır. Alt çenede, implant cerrahisi öncesi kret tepesinden mandibular kanala olan mesafenin ölçülmesi rutin yapılan bir hazırlıktır. Bunun yanında koronal düzlemde mandibulanın anatomik yapısının değerlendirilmesi bu yapılamıyorsa ameliyat sırasında kret morfolojisinin palpasyonla muayenesi ameliyat sırasında ve sonrasında gelişebilecek komplikasyonların önlenmesi açısından önem arz etmektedir.

\section{ANAHTAR KELIMELER}

Alt çene, diş implantı, konik ışınlı bilgisayarlı tomografi, sublingual kanama
ABSTRACT

Risk assessment of lingual plate perforation in posterior mandibular region during implant placement: A retrospective study using 3D virtual implant placement program

Background: The aim of this study was to determine the risk of lingual perforation related with structure of the crest and evaluate the prevalence of lingual perforation in virtually inserted dental implants to premolar and molar area with the using of computer aided planning program on cone beam computerized tomography (CBCT) images.

Methods: CBCT images of 543 patients from the archives of Izmir Katip Çelebi University, Faculty of Dentistry were scanned and 179 implants were virtually inserted on CBCT images of 107 patients who met inclusion criteria using a virtual implant placement program (Simplant $\left.{ }^{\circledR}\right)$. Crests were classified according to sections as $\mathrm{P}, \mathrm{C}$ and $\mathrm{U}$ types. Proximity of lingual plate to implants, inserted $2 \mathrm{~mm}$ apart from inferior alveolar canal, was evaluated.

Results: The majority of lingual perforations were seen in $U$ type crests $(\% 87,5)$ and risk of perforation tends to increase after $8 \mathrm{~mm}$ of length. Four (\% 8,3) perforations were encountered in C-type crests and lingual perforation was seen in only two $(\% 4,2)$ patients in $14 \mathrm{~mm}$ length in P-type crests.

Conclusion: The results of this study once again demonstrated the importance of CBCT in assessing the proximity of anatomical structures to the area of osteotomy in implant applications. The measurement of the distance from the alveolar crest to the mandibular canal is a routine pre-implant surgery preparation. Mandibular anatomic structure should be assessed on coronal plane, if it's not possible, examination with palpation of crest structure is important in prevention of the pre-operative and postoperative complications.

\section{KEYWORDS}

Mandible, dental implant, cone beam computerized tomography, sublingual bleeding

\footnotetext{
${ }^{\alpha}$ İzmir Katip Çelebi Üniversitesi Diş Hekimliği Fakültesi Ağız Diş ve Çene Cerrahisi AD, İzmir

${ }^{\beta}$ Serbest diş hekimi

${ }^{v}$ İzmir Katip Çelebi Üniversitesi Diş Hekimliği Fakültesi Ağız Diş ve Çene Radyolojisi AD, İzmir

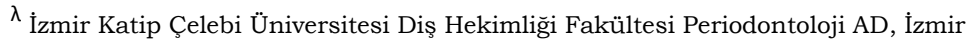


Dental implant tedavisi oral cerrahi uygulamalar arasında, yüksek başarı oranıyla önemli bir yer tutmaktadır. Geniş dişsiz boşlukların rehabilitasyonundan tek diş eksikliklerinin giderilmesine kadar birçok farklı vakada sıklıkla uygulanan dental implant tedavisiyle kaybedilen fonksiyonun ve estetiğin hastalara geri kazandırılması hedeflenmektedir. ${ }^{1,2,3}$

İmplant cerrahisinden önce protetik restorasyonun final pozisyonu belirlenmeli, tasarlanan proteze optimum destek sağlayacak konumda dental implant çene kemiği içerisine yerleştirilmelidir. ${ }^{4}$ İmplantın ideal pozisyonda yerleştirilmesi uzun dönem başarı açısından oldukça önemlidir. ${ }^{2}$ İmplantı istenilen pozisyonda yerleştirmek, anatomik oluşumların sebep olduğu kısıtlamalara bağlı olarak, her zaman mümkün olmayabilir. Bazı vakalarda mandibulada, özellikle posterior bölgede, lingualde iç bükeylik görülmekte ve bu anatomik durum implant cerrahisini güçleştirebilmektedir. Watanabe ve ark. $^{5}$ mandibulanın kesitsel morfolojisini kategorize ettikleri çalışmalarında populasyonun \% 36-39'unda lingual bölgede konkavite görüldüğünü rapor etmişlerdir. İmplantın komşu dişlerin uzun aksına ve karşıt dişlerin okluzal düzlemine uygun mesiodistal ve bukkolingual açıda yerleştirilmesi biyomekanik olarak ideal olmasına rağmen, lingual iç bükeylik dikkate alınmadığında lingual plakta perforasyonlar oluşabilmektedir. Aynı şekilde, anatomik sınırlar dikkate alınmadan manuel olarak hazırlanan cerrahi rehberlerin kullanımı da birtakım cerrahi komplikasyonlara yol açabilmektedir. ${ }^{6}$

Konik ışınlı bilgisayarlı tomografi (KIBT), implant cerrahisi öncesi tedavi planlamasında komplikasyonları en aza indirip daha başarılı sonuçlar elde etmek amacıyla yaygın olarak kullanılmaktadır. $^{7}$ KIBT görüntüleri üzerinde, farklı firmaların geliştirdiği yazılım programları kullanılarak dental implantların ideal pozisyonları ve açıları ameliyat öncesi sanal ortamda belirlenebilmekte ve elde edilen bu veriler ışığında son derece hassas cerrahi rehber plaklar üretilebilmektedir. ${ }^{8,9}$

Literatürde, implant konumunun belirlenmesinde kemik morfolojisinin, özellikle de lingual iç bükeyliğin etkisinin değerlendirildiği ve hatalı pozisyonda yerleştirilen implantların neden olduğu lingual perforasyon prevelansının araştırıldığı yeterince çalışma bulunmamaktadır. Bu çalışmanın amacı; bilgisayar yazılımı kullanarak KIBT görüntüleri üzerinde, dişsiz mandibular molar ve premolar bölgelere protetik olarak ideal konumda yerleştirilen implantların olası lingual perforsayon prevelansını değerlendirmek ve kretin morfolojik yapısı ile lingual kortikal kemik perforasyon riski arasındaki ilişkiyi belirlemektir.

\section{GEREÇ VE YÖNTEM}

Bu çalışma, 2015 Ocak - 2016 Kasım tarihleri arasında İzmir Kâtip Çelebi Üniversitesi Diş Hekimliği Fakültesi'ne farklı şikâyetlerle başvuran, tedavileri esnasında ortopantomografi (OPG) ve KIBT çekilen hastalara ait veriler kullanılarak planlanmıştır. OPG ve KIBT çekilen 543 hastaya ait, artefakt bulunmayan fakülte arşivindeki radyolojik görüntüler çalışma amacı ile taranmıştır.

Çalışmaya dahil edilme kriterleri aşağıda belirtilmiştir;

1) Posterior mandibular premolar ve molar bölgeye ait OPG ve KIBT görüntüsü mevcut olan,

2) Posterior mandibular ikinci premolar, birinci ve ikinci molar dişlerden en az bir tanesini kaybetmiş olan,

3) İmplantın uygun pozisyon ve açıda yerleştirilmesi için, eksik olan diş bölgesinin karşıt arkında okluzyonu sağlayabilecek dişi mevcut olan,

4) Dişsiz bölgenin komşuluğundaki dişleri normal pozisyonda olan,

5) Implant yerleştirilecek olan posterior mandibular bölgede inferior alveolar kanalın üst sınırı ile kret tepesi arasında en az $10 \mathrm{~mm}$ kemik yükseliği olan,

6) Posterior mandibular bölgede, horizontal düzlemde yeterli kemik genişliğine sahip olan hastalara ait radyolojik görüntüler çalışmaya dâhil edilmiştir.

Çalışmaya dâhil edilmeme kriterleri aşağıda belirtilmiştir;

1) Posterior mandibulada geniş patolojik oluşumları bulunan,

2) İmplant yerleştirilecek bölgede gömülü dişleri bulunan,

3) Komşuluktaki mandibular dişleri normal pozisyonda olmayan,

4) Karşıt arkta, okluzyonu kontrol edebilmek amaciyla dişleri bulunmayan hastalara ait radyolojik görüntüler çalışmaya dahil edilmemiştir.

Değerlendirmeye alınan 543 hastaya ait KIBT görüntülerinden kriterlere uyan 107 tanesi çalışmaya dahil edilmiştir. Çalışmaya dahil edilen hastaların 39'u erkek 68'i kadın ve yaş aralığı 18 - 61 'dir (ortalama 38,54). 107 hastada toplam 179 implant, Simplant ${ }^{\circledR}$ (Materialise, Belçika) implant planlama programı kullanılarak dişsiz mandibular premolar ve molar bölgelere sanal ortamda yerleştirilmiştir. 145 implant birinci ve ikinci molar bölgesine, 34 implant ise ikinci premolar bölgesine yerleştirilmiştir.

Yerleştirilecek olan implantların uzunluğu deneyimli bir cerrah tarafından komşu dişlerden yaklaşık $1,5 \mathrm{~mm}$, inferior alveolar kanalın üst sınırından en az $2 \mathrm{~mm}$ olacak şekilde OPG üzerinde ölçümü yapılarak belirlendi. Daha sonra belirlenen değerlere göre 
implantlar Simplant ${ }^{\circledR}$ programında ilgili bölgelere sanal ortamda, bukkal ve lingual kortikal kemiklerden en az $1 \mathrm{~mm}$ uzakta olmak kaydıyla aksiyal kesitte kretin bukkolingual olarak ortasında olacak şekilde ve komşu dişlerin santral fossalarından geçen hayali çizgi üzerinde olacak şekilde yerleştirilmiştir (Resim 1).
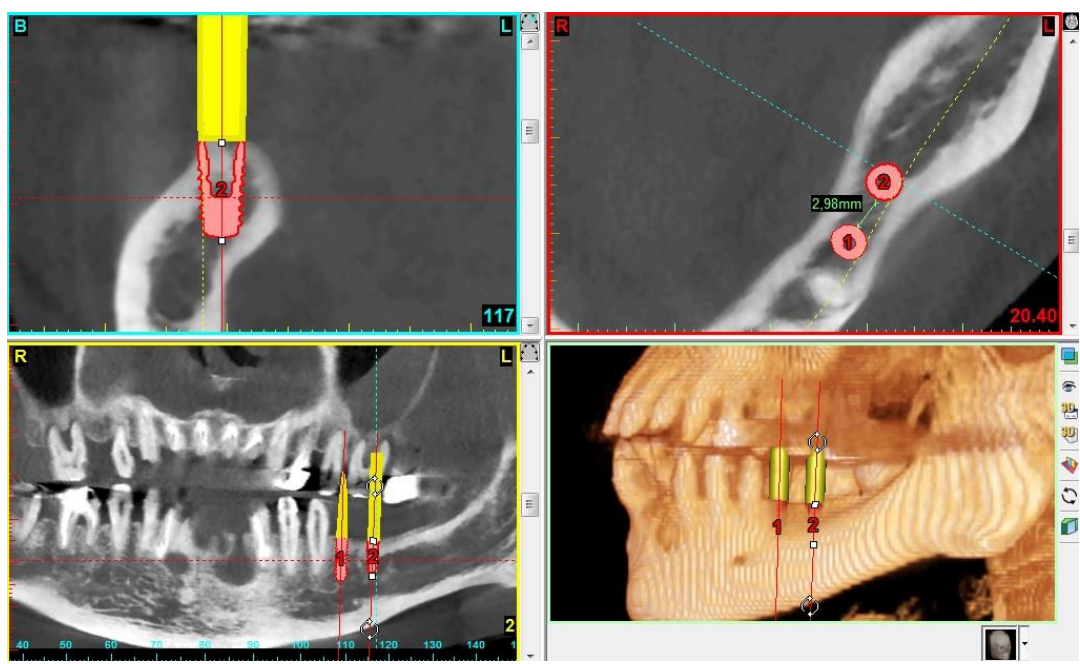

Resim 1.

Simplant ${ }^{\circledR}$ programında KIBT görüntüsü üzerinde dental implantların konumlandırılması

Yine karşıt arkta bulunan maksiller dişlerin bukkal kasplarının, yerleştirilen implant ile olan ilişkileri de protetik açıdan değerlendirilmiştir. Yan yana yerleştirilen implantların birincisi yukarıda bahsedilen kriterler göz önünde bulundurularak yerleştirildikten sonra diğer implant(lar) da bu implanta paralel olacak şekilde ve yukarıda bahsedilen diğer kriterlere uyularak yerleştirilmiş ve implantların apikali ile lingual kortekse olan mesafeleri ölçülerek kaydedilmiştir.

Çalışmada posterior dişsiz mandibuladaki kret tipleri Chan ve ark. ${ }^{2}$ tarafından tanımlanan şekilde sınıflandırılmıştır. Koronal kesitlerde bukkal ve lingual kortikal kemiğin paralel olduğu kret tipleri P tipi, lingualde balkon olan ve andırkat mevcut olan kretler $U$ tipi ve alveolar kanala doğru genişleyenler ise C tipi olarak sınıflandırılmıştır (Resim 2). Buna göre çalışmamızda yerleştirilen implantların 78'i U tipi krete, 63'ü C tipi krete, 38'i P tipi krete yerleştirilmiştir. Yerleştirilen bu implantların 48'inde $(\% 26,8)$ lingualden perforasyon ya da çok yakın ilişki belirlenmiştir. Lingualden perfore olan vakalarda implantların 42 tanesi $U$ tipi krete, 2 tanesi $P$ tipi krete ve 4 tanesi de C tipi krete yerleştirilmiştir
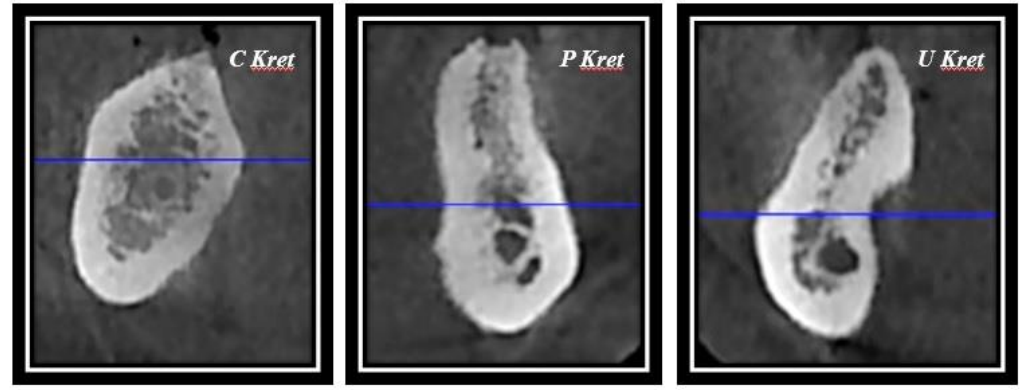

Resim 2.

Koronal düzlemde posterior mandibulanın morfolojisi (Mavi çizgi, inferior alveolar kanalın 2 mm üzerinden geçmektedir)

\section{İstatistiksel analiz}

İstatistiksel analizler SPSS (IBM Co., Chicago, IL, ABD) yazılımı kullanılarak yapılmıştır.

Perforasyonların kretlere göre dağılım yüzdeleri, lingual kemik perforasyonlarının implant uzunluklarına/gruplara göre dağılımı ve implant uzunluğu dikkate alınarak kret tipi ile perforasyon ilişkilerinin değerlendirilmesinde Ki-kare testi kullanılmıştır. Perforasyon, kret tipi ve implant uzunlukları tablosunda beşten küçük beklenen değerlerin bulunduğu hücre sayısı \%20'nin üzerinde bulunduğu için uygun modelleme ikişerli Ki-kare tabloları sonuçlarına göre yapılmıştır. İstatistiksel anlamlılık düzeyi $p \leq 0,05$ olarak belirlenmiştir.

\section{BULGULAR}

Dahil edilme kriterlerini karşılayan, yaş ortalaması 38,5 (18-61) olan 39 (\% 36,4) erkek ve 68 (\% 63,6) kadın olmak üzere toplamda 107 hastada 179 sanal implant uygulaması yapılmıştır. Perforasyonların kretlere göre dağılım yüzdeleri ve istatistiksel karşılaştırmaları Tablo 1'de verilmiştir. \%21 P tipi (38), \%35 C tipi (63) ve \%44 U tipi (78) olmak üzere toplam 179 kret incelenmiştir.

Lingual kemik perforasyonlarının, implant uzunlukları ve kret tipleri ile ilişkisi Tablo 2'te sunulmuştur. Kret tipi ile perforasyon arasındaki ilişki istatistiksel olarak anlamlı bulunurken $(p<0.001)$; kret tipi-implant uzunluğu veya perforasyon-implant uzunluğu arasındaki farklar istatistiksel olarak anlamlı değildir.

Tablo 3'te, implant uzunluğu dikkate alınarak, kret tipi ile perforasyon ilişkileri incelenmiştir. Buna göre $8 \mathrm{~mm}$ uzunluğunda implant seçildiğinde kret tipine göre perforasyon olasılığı değişmemektedir. 10 ve $12 \mathrm{~mm}$ implantlar seçildiğinde, U tipi krette perforasyon riski $\mathrm{C}$ tipine göre daha yüksek bulunmuş, diğer gruplar arasında fark bulunmamıştır. $14 \mathrm{~mm}$ implant seçildiğinde, $U$ tipi krette perforasyon riski $\mathrm{C}$ ve $\mathrm{P}$ tiplerine göre daha yüksek bulunmuş, diğer gruplar arasında fark gözlenmemiştir. 
Tablo 1.

$\begin{aligned} & \text { Perforasyonların kretlere göre dağılım } \\ & \text { yüzdeleri }\end{aligned}$
a

\begin{tabular}{|c|c|c|c|c|c|}
\hline \multirow[b]{2}{*}{ Perforasyon } & \multicolumn{4}{|c|}{ Kret tipi } & \multirow[b]{2}{*}{$p$} \\
\hline & $\begin{array}{c}P \\
\text { n (\%) }\end{array}$ & $\begin{array}{c}\text { C } \\
\text { n (\%) }\end{array}$ & $\underset{\text { n (\%) }}{U}$ & Toplam & \\
\hline Yok & $\begin{array}{c}36 \\
(94.7)\end{array}$ & $\begin{array}{c}59 \\
(93.7)\end{array}$ & $\begin{array}{c}36 \\
(46.2)\end{array}$ & 131 & \multirow{2}{*}{$<0.001$} \\
\hline Var & $\begin{array}{c}2 \\
(5.3)\end{array}$ & $\begin{array}{c}4 \\
(6.3)\end{array}$ & $\begin{array}{c}42 \\
(53.8)\end{array}$ & 48 & \\
\hline Toplam & 38 & 63 & 78 & 179 & \\
\hline
\end{tabular}

Tablo 2

Lingual kemik perforasyonları, implant uzunlukları ve kret tipleri arasındaki ilişki

\begin{tabular}{|lccc|} 
& $\chi^{2}$ & sd & p \\
\hline Kret tipi X Perforasyon & 51.481 & 2 & $<0.001$ \\
\hline $\begin{array}{l}\text { Kret tipi X İmplant Uzunluğu } \\
\begin{array}{l}\text { Perforasyon X İmplant } \\
\text { Uzunluğu }\end{array}\end{array}$ & 10.878 & 6 & 0.090 \\
\hline
\end{tabular}

Tablo 3.

İmplant uzunluğu dikkate alınarak kret tipi ile perforasyon ilişkileri

\begin{tabular}{|c|c|c|c|c|c|c|}
\hline \multirow{2}{*}{$\begin{array}{l}\text { İmplant } \\
\text { Uzunluğu }\end{array}$} & \multirow[b]{2}{*}{ Perforasyon } & \multicolumn{3}{|c|}{ Kret tipi } & \multirow{2}{*}{ Toplam } & \multirow{2}{*}{$p$} \\
\hline & & $P$ & C & $\mathbf{U}$ & & \\
\hline \multirow{2}{*}{$8 \mathrm{~mm}$} & Yok & 2 & 3 & 4 & 9 & \multirow{2}{*}{1000} \\
\hline & Var & 0 & 0 & 1 & 1 & \\
\hline \multirow{2}{*}{$10 \mathrm{~mm}$} & Yok & 3 & 17 & 8 & 28 & \multirow{2}{*}{0.003} \\
\hline & Var & $0^{a b}$ & $0^{a}$ & $8^{b}$ & 8 & \\
\hline \multirow{2}{*}{$12 \mathrm{~mm}$} & Yok & 6 & 14 & 11 & 31 & \multirow{2}{*}{0.002} \\
\hline & Var & $0^{a b}$ & ${ }_{1}^{a}$ & $12^{b}$ & 13 & \\
\hline \multirow{2}{*}{$14 \mathrm{~mm}$} & Yok & 25 & 25 & 13 & 63 & \multirow{2}{*}{$<0.001$} \\
\hline & Var & $2^{a}$ & $3^{a}$ & $21^{b}$ & 36 & \\
\hline \multicolumn{2}{|c|}{ Toplam } & 38 & 63 & 78 & 179 & \\
\hline
\end{tabular}

\section{TARTIŞMA}

İmplant cerrahisi öncesi ameliyat bölgesinin KIBT ile değerlendirilmesi son derece önemlidir. Çene kemiklerinin morfolojisi değerlendirilerek, önemli anatomik yapıların implant yapılması planlanan bölgeye uzaklığı tespit edilip cerrahi işlem sırasında ve sonrasında yaşanabilecek komplikasyonların önüne geçilebilir. Alt çenede, özellikle posterior mandibulada implant cerrahisi planlanıyorsa KIBT görüntüleri üzerinde ilgili bölgenin morfolojisi dikkatlice incelenmelidir. İnferior alveolar sinirin kret tepesine yakın seyretmesi ya da lingual bölgenin iç bükey yapıda olması gibi anatomik varyasyonlar önceden tespit edilmez ise istenmeyen komplikasyonlarla karşılaşılabilir. ${ }^{10,11}$ Posterior mandibular bölgede lingual konkavitenin varlığında lingual kemik korteksinin perforasyon riski nedeniyle osteotomi esnasında daha dikkatli çalışılması gerekmektedir. ${ }^{12}$ Bu çalışmada, mandibulanın aksiyal ve koronal düzlemlerindeki morfolojisi ile implant cerrahisi sırasında lingual kemikte perforasyon meydana gelme intimali arasındaki korelasyon incelendi. Chan ve ark. ${ }^{2}$ yapmış oldukları araştırmada çalışma grubunu oluşturan hastalar koronal düzlemde \% $13.6 \cup$ (undercut), \% 20,4 C (konverjant), \% $66 \mathrm{P}$ (paralel) tipi kret morfolojisine sahipken çalışmamızda sırasıyla kret tipi oranları \% 44, $\% 35$ ve \% 21 şeklinde bulunmuştur. Parnia ve ark..$^{13}(\%$ $80)$, Huang ve ark. ${ }^{14}(\% 56,2)$ ile Nickening ve ark. ${ }^{15}(\%$ 56) yapmış oldukları çalışmalarda, çalışmamızdaki verilere benzer şekilde, U tipi kret varlığının daha yüksek olduğunu tespit etmişlerdir.

Kretin morfolojisi, yerleştirilen implantın çapı ve uzunluğu ameliyat sırasında lingual kemikte perforasyon meydana gelme intimalini doğrudan etkileyen faktörlerdir. Yapılan çalışmalar $U$ tipi krette perforasyon meydana gelme ihtimalinin $C$ ve $P$ tipine göre daha yüksek olduğunu göstermiştir. 2,14 Aynı zamanda implantın uzunluğu arttıkça lingual kortikal kemikte perforasyon riski, özellikle $U$ tipi krette, artacaktır. $\mathrm{Bu}$ çalışmanın sonuçlarına göre her üç kret tipinde toplam implant/perforasyon oranı $\% 26,8$ bulunmuştur. Aynı zamanda lingual plakta oluşan perforasyonların \% 87,5 'inin U tipi krette meydana geldiği görülmüştür. Benzer şekilde Huang ve ark.14 en yüksek perforasyon intimalinin \%87 ile U tipi krette olduğunu göstermişlerdir.

İmplant cerrahisi sırasında hatalı uygulamalara bağlı oluşan sublingual hemoraji vakalarına literatürde rastlamak mümkündür. $\mathrm{Bu}$ vakalarda, özellikle interforaminal bölgede yapılan implant yuvası hazırlama işlemi sırasında hekim hatası nedeniyle lingual kemikte perforasyon oluştuğu ve bunun neticesinde sublingual arterlerin hasarına bağlı, hayatı tehdit edecek düzeyde solunum obstrüksiyonuna kadar gidebilen, sublingual hemoraji meydana geldiği görülmektedir. ${ }^{16,17,18}$ Benzer komplikasyonun posterior mandibulada yapılan implant cerrahisi sırasında meydana gelmesi olasıdır. Literatürde, molar bölgeye implant yerleştirildikten hemen ya da birkaç saat sonra şiddetli sublingual hemorajinin oluştuğunu bildiren raporlar mevcuttur. ${ }^{19,20}$ Dakikalar içerisinde ağız tabanının yükselmesine neden olan, vasküler (sublingual arter/ ven, submental arter/ven) hasara bağlı oluşan kanamalar gerekli müdahale yapılmaz ise hayatı tehdit edici boyuta ulaşabilmektedir. 
İmplant cerrahisi öncesi planlama yaparken, çene kemiğinin morfolojisini ve önemli anatomik yapıların lokalizasyonunu belirlemede KIBT altın standart olarak kabul edilmektedir. Ancak birçok klinikte bu görüntüleme tekniğine ulaşım mümkün olmayıp, OPG üzerinde 2 boyutlu değerlendirme yapılmaktadır. Bu yöntemde implant yapılacak bölge ancak vertikal düzlemde değerlendirilebilmektedir. 3 boyutlu değerlendirmenin yapılamadığı vakalarda, cerrahiden hemen önce hasta klinik olarak çok dikkatli değerlendirilmeli, özellikle alt çenede implant yapılacak bölgenin lingual bölümü ağız tabanına doğru iç bükeylik açısından, palpasyonla özenle muayene edilmelidir. Bazı çalışmalar ameliyat öncesi lingual bölgenin prob yardımıla muayene edilmesi ya da lingual mukoperiosteal flebin tümüyle kaldırılarak bölgenin anatomisinin gözlemlenmesini tavsiye etmektedir. ${ }^{21,22}$

\section{SONUÇ}

$\mathrm{Bu}$ çalışmanın sonuçları dikkate alındığında "U" tipi kretlerde $8 \mathrm{~mm}$ uzunluktan sonra lingual perforasyon intimalinin anlamlı şekilde arttığı, $12 \mathrm{~mm}$ uzunluktan sonra perforasyon intimalinin çok yüksek olduğu görülmektedir. OPG değerlendirmesinde inferior alveolar kanala yeterli mesafe olduğunda, posterior mandibulada 12 mm'den daha uzun implant kullanılacaksa azami dikkat gösterilmelidir.

$\mathrm{Bu}$ çalışmanın sonuçları, literatürde yer alan birçok çalışma gibi implant uygulamalarında rutin olarak KIBT kullanımının gerekliliğine işaret etmektedir. Lingual iç bükeylik ile lingual perforasyon riski arasındaki ilişkinin değerlendirilmesinde daha fazla çalışmanın yapılmasına intiyaç duyulmaktadır.

\section{Teşekkür}

Verilerin istatistiksel analizinde yardımcı olan sayın Doç. Dr. Ferhan ELMALI'ya teşekkür ederiz. 


\section{KAYNAKLAR}

1. Scheller H, Urgell JP, Kultje C, Klineberg I, Goldberg $\mathrm{PV}$, Stevenson-Moore P, et al. A 5-year multicenter study on implant-supported single crown restorations. Int J Oral Maxillofac Implants 1998; 13: 212-8.

2. Chan HL, Benavides E, Yeh $\mathrm{CY}, \mathrm{Fu} \mathrm{JH}$, Rudek IE, Wang HL. Risk Assessment of Lingual Plate Perforation in Posterior Mandibular Region: A Virtual Implant Placement Study Using Cone-Beam Computed Tomography. J Periodontol 2011; 82: 129-35.

3. Adell R, Lekholm U, Rockler B, Branemark PI. A 15year study of osseointegrated implants in the treatment of the edentulous jaw. Int J Oral Surg 1981; 10: 387416.

4. Becker CM, Kaiser DA. Surgical guide for dental implant placement. J Prosthet Dent 2000; 83: 248-51.

5. Watanabe $\mathrm{H}$, Mohammad Abdul M, Kurabayashi $\mathrm{T}$, Aoki $\mathrm{H}$. Mandible size and morphology determined with CT on a premise of dental implant operation. Surg Radiol Anat 2010; 32: 343-9.

6. White SC. Cone-beam imaging in dentistry. Health Phys 2008; 95: 628-37.

7. Ganz SD. Computer-aided design/computer-aided manufacturing applications using CT and cone beam CT scanning technology. Dent Clin North Am 2008; 52 : 777-808.

8. Schneider D, Marquardt P, Zwahlen M, Jung RE. A systematic review on the accuracy and the clinical outcome of computer-guided template-based implant dentistry. Clin Oral Implants Res 2009; 20(Suppl. 4): 73-86.

9. van Assche N, van Steenberghe D, Guerrero ME, et al. Accuracy of implant placement based on pre-surgical planning of three-dimensional cone-beam images: A pilot study. J Clin Periodontol 2007; 34: 816-21

10. Froum S, Casanova L, Byrne S, Cho SC. Risk assessment before extraction for immediate implant placement in the posterior mandible: a computerized tomographic scan study. J Periodontol. 2011; 82(3): 395-402.

11. Chan HL, Brooks SL, Fu JH, Yeh CY, Rudek I, Wang $\mathrm{HL}$. Crosssectional analysis of the mandibular lingual concavity using cone beam computed tomography. Clin Oral Implants Res 2011; 22(2): 201-6.

12. Greenstein G, Cavallaro J, Tarnow D. Practical application of anatomy for the dental implant surgeon. J Periodontol 2008; 79: 1833-46.

13.Parnia F, Fard EM, Mahboub F, Hafezeqoran A, Gavgani FE. Tomographic volume evaluation of submandibular fossa in patients requiring dental implants. Oral Surg Oral Med Oral Pathol Oral Radiol Endod. 2010; 109: 32-6.

14. Huang RY, Cochran DL, Cheng WC, Lin MH, Fan WH, Sung $C E$, et al. Risk of lingual plate perforation for virtual immediate implant placement in the posterior mandible. JADA 2015;146(10):735-42.
14.Nickenig HJ, Wichmann M, Eitner S, Zöller JE, Kreppel M. Lingual concavities in the mandible: a morphological study using crosssectional analysis determined by CBCT. J Craniomaxillofac Surg 2015; 43: 254-9.

15.Dubois L, de Lange J, Baas E, Van Ingen J. Excessive bleeding inthe floor of the mouth after endosseus implant placement: a report oftwo cases. Int J Oral Maxillofac Surg 2010; 39: $412-5$.

16. Givol N, Chaushu G, Halamish-Shani T, Taicher S. Emergencytracheostomy following life-threatening hemorrhage in the floor ofthe mouth during immediate implant placement in the mandibularcanine region. J Periodontol 2000; 71: 1893-5.

17.Isaacson TJ. Sublingual hematoma formation during immediate placement of mandibular endosseous implants. J Am Dent Assoc 2004;135:168-72.

18. Ferneini, E, Gady, J, Liebrich, SE. Floor ofthe mouth hematoma after poste rior mandibular implants placement: a case report. Journal of Oral and Maxillofacial Surgery 2009; 67: 155254

19.Del Castillo-Pardo de Vera, JL, Lopez-Arcas Call-eja, JM, Burgueno-Garcia, M. Hematoma of the floor of the mouth and airway obstruction during mandibular dental implant placement: a case report. J Oral Maxillofac Surg 2008; 12: 223-6.

20. Hofschneider U, Tepper G, Gahleitner A, UIm C. Assessment of the blood supply to the mental region for reduction of bleeding complications during implant surgery in the interforaminal region. Int $\mathrm{J}$ Oral Maxillofac Implants 1999; 14: 379-83.

21.Kalpidis CD, Setayesh RM. Hemorrhaging associated with endosseous implant placement in the anterior mandible: a review of the literature. J Periodontol 2004; 75: 631-45.

\section{Yazışma Adresi:}

Murat ULU

İzmir Katip Çelebi Üniversitesi

Diş Hekimliği Fakültesi

Ağız Diş ve Çene Cerrahisi AD

Cemil Meriç Cd. 6780 Sk. No:48

35640, Çiğli, İzmir

Tel : +902323254040/2250

GSM : +90542673 8078

E-mail: murat.ulu@ikc.edu.tr muratulu81@hotmail.com 